\title{
Characteristics of culprit lesions in young patients with metabolic syndrome and classic cardiovascular risk factors
}

\author{
FANGJIE HOU ${ }^{1,2}$, YUJIE ZHOU ${ }^{2}$, WEI LIU ${ }^{2}$, SHIWEI YANG ${ }^{2}$, ZHIJIAN WANG $^{2}$, \\ XIAOTENG MA ${ }^{2}$, YU DU ${ }^{2}$, YA LI $^{2,3}$ and JUN GUAN ${ }^{1}$ \\ ${ }^{1}$ Department of Cardiology, Qingdao Municipal Hospital, Qingdao, Shandong 266000; \\ ${ }^{2}$ Department of Cardiology, Beijing Anzhen Hospital, Capital Medical University, Beijing 100029; \\ ${ }^{3}$ Department of Cardiology, Affiliated Hospital of Hebei University, Baoding, Hebei 071000, P.R. China
}

Received June 25, 2019; Accepted January 6, 2020

DOI: $10.3892 /$ etm.2020.8484

\begin{abstract}
The association between cardiovascular risk factors (CVRFs) and characteristics of coronary plaque in young patients has remained to be fully elucidated. Therefore, the present study sought to determine the association between CVRFs and phenotypes of culprit coronary plaques revealed by optical coherence tomography (OCT) in young patients with stable coronary heart disease (CHD) and acute coronary syndrome (ACS). OCT imaging pullback was performed at corresponding sites on 123 lesions in 123 young patients (age, 36 \pm 7 years), including those with stable CHD and ACS. Patients with analyzable OCT images were classified as having thin-cap fibroatheromas (TCFAs), plaque rupture, macrophage accumulation, calcified nodule, vasa vasorum, cholesterol crystal and erosion. TCFAs were more prevalent in patients with metabolic syndrome (MetS) than in those without MetS $(\mathrm{P}=0.020)$. Plaque rupture was more common in smokers than in non-smokers $(\mathrm{P}=0.002)$. Multivariate analysis indicated that MetS was independently associated with TCFAs $(\mathrm{P}=0.041)$ and smoking was independently associated with plaque rupture $(\mathrm{P}=0.006)$. Young patients with MetS were demonstrated to have more extensive TCFAs and young smokers had a higher prevalence of culprit plaque rupture.
\end{abstract}

\section{Introduction}

Coronary heart disease (CHD) is correlated with well-acknowledged cardiovascular risk factors (CVRFs), including cigarette

Correspondence to: Dr Jun Guan, Department of Cardiology, Qingdao Municipal Hospital, 1 Jiaozhou Road, Qingdao, Shandong 266000, P.R. China

E-mail: guanjun3582@163.com

Dr Yujie Zhou, Department of Cardiology, Beijing Anzhen Hospital, Capital Medical University, 2 Anzhen Road, Beijing 100029, P.R. China

E-mail: azzyj12@163.com

Key words: vulnerable plaque, optical-coherence tomography, premature coronary heart disease, coronary artery disease, cardiovascular risk factors smoking, type 2 diabetes (T2D), hypertension, hypercholesterolemia, family history and metabolic syndrome (MetS) $(1,2)$. Imaging studies have demonstrated an association between plaque phenotypes and CVRFs in middle-aged and elderly patients with CHD (3-5). However, the pathophysiology of atherosclerosis in young patients with CHD differs from that in older patients (6). To date, the association between CVRFs and the characteristics of culprit coronary plaque in young patients has remained to be fully elucidated. Furthermore, the incidence of CHD has increased in young individuals. CHD may have serious consequences, including premature death and long-term disability (7).

Optical coherence tomography (OCT) has emerged as the most accurate imaging modality for intracoronary evaluation, with a resolution of $10-20 \mu \mathrm{m}$ (8). OCT has been widely used to investigate atherosclerotic plaque microstructure, which may be a key factor in determining plaque stability (9). OCT findings are validated by histologic evaluation (10). In the present study, the association between the phenotype of the culprit atherosclerotic plaque as determined by OCT and CVRFs in young patients were assessed.

\section{Patients and methods}

Patients. The present study was a retrospective, single-center study. Consecutive patients (age, $36 \pm 7$ years; male $87 \%$, female 13\%) who underwent OCT between April 2014 and March 2017 in the Cardiology Department of Beijing Anzhen Hospital, including those with stable CHD and acute coronary syndrome (ACS), were selected. The exclusion criteria were a known history of severe hepatic or renal dysfunction, an ongoing inflammatory condition, familial hypercholesterolemia and arthritis. Patients with poor image quality, incomplete pullback, or missing data were also excluded. All of the patients provided informed consent and the study protocol was approved by the Ethics Committee of the Beijing Anzhen Hospital (Beijing, China).

Definition of CVRFs. The definition of MetS was based on the criteria established in the Joint Scientific Statement (11). An adult with $\geq 3$ of the following was deemed to have MetS: Waist circumference, $\geq 90 \mathrm{~cm}$ for males or $\geq 80 \mathrm{~cm}$ for females; triglycerides, $\geq 150 \mathrm{mg} / \mathrm{dl}$; high-density lipoprotein cholesterol, 
$\leq 40 \mathrm{mg} / \mathrm{dl}$; systolic blood pressure (SBP), $\geq 130 \mathrm{mmHg}$ and/or diastolic blood pressure (DBP), $\geq 85 \mathrm{mmHg}$, or treated hypertension; and fasting blood glucose level, $\geq 100 \mathrm{mg} / \mathrm{dl}$ or treated T2D. Smoking was defined as current cigarette smoking. Hypertension was defined as SBP $\geq 140 \mathrm{mmHg}$ and/or DBP $\geq 90 \mathrm{mmHg}$, or treated hypertension. T2D was defined as fasting blood glucose $>126 \mathrm{mg} / \mathrm{dl}$ or treated T2D (a diabetic diet or prescription of oral hypoglycemic agent). Hypercholesterolemia was defined as total cholesterol $>200 \mathrm{mg} / \mathrm{dl}$ or treated hypercholesterolemia. A family history of coronary artery disease (CAD) was defined as premature $\mathrm{CAD}$ in a first-degree relative (a male aged $<55$ years or a female aged $<65$ years).

Coronary angiography (CAG) and OCT procedures. Diagnostic angiograms were recorded via radial access using a 5.24-mm French (5-Fr) catheter and after administering a 5,000-IU bolus of heparin. Culprit lesions were identified via CAG and electrocardiographic ST-segment alterations. The decision of whether to perform OCT was at the discretion of the operator. A 0.014 -inch guidewire was placed distally in the target vessel and an intracoronary injection of $200 \mathrm{mg}$ nitroglycerin was administered via a 6-Fr guiding catheter. Frequency domain OCT images were acquired using a C7-XR OCT Intravascular Imaging System (St. Jude Medical), which was advanced to the culprit lesion. During image acquisition, the coronary blood flow was replaced by continuously flushing contrast media directly from the guiding catheter at a rate of 3-4 ml/sec with a power injector, thus creating a virtually blood-free environment with the integrated automated pullback device at $20 \mathrm{~mm} / \mathrm{sec}$. In the OCT investigations, 5-10 ml of contrast media was used, the flouro time was $2-4 \mathrm{sec}$ and the radiation dose was 30.6-61.2 mGray.

OCT image analysis. The operator who performed the pullback and an independent investigator who was blinded to the clinical presentation analyzed the OCT images offline. Any disagreements were resolved by consensus. A thin-cap fibroatheroma (TCFA) was defined as an OCT-delineated necrotic core subtending a $>90^{\circ}$ arc and covered by a fibrous cap with a thickness of $<65 \mu \mathrm{m}$ (5). Plaque erosion was defined by the presence of preserved vascular integrity (intact fibrous cap), a larger residual lumen and a platelet-rich thrombus (12). A vasa vasorum was defined as a small black hole within a plaque, 50-300 $\mu \mathrm{m}$ in diameter, that was present on at least 3 consecutive frames in pullback images (13). Cholesterol crystals were defined as thin linear structures with high backscatter and without attenuation within the plaque (14). Plaque rupture, macrophage accumulation, calcified nodules and percent area stenosis (AS\%) were defined as per the International Working Group for Intravascular Optical Coherence Tomography consensus standards (15).

Statistical analysis. Categorical data are presented as counts and proportions and were compared using a $\chi^{2}$ test. The distributions of the continuous variables across the study groups were tested with the Shapiro-Wilks test. Normally distributed data are presented as the mean \pm standard deviation and were compared using an independent-samples t-test. Non-normally distributed data are presented as the median (interquartile range)
Table I. Baseline characteristics of the patients $(n=123)$.

\begin{tabular}{|c|c|}
\hline Item & Value \\
\hline Age (years) & $36 \pm 7(20-45)$ \\
\hline Male sex & $107(87.0)$ \\
\hline Family history of CHD & $10(8.1)$ \\
\hline Smoking & $67(54.5)$ \\
\hline Hypertension & $63(51.2)$ \\
\hline Diabetes mellitus & $22(17.9)$ \\
\hline Hypercholesterolemia & $15(12.2)$ \\
\hline Metabolic syndrome & $82(66.7)$ \\
\hline $\mathrm{ACS}$ & $77(62.6)$ \\
\hline Smoking & $49(63.6)$ \\
\hline Stable CHD & $46(37.4)$ \\
\hline Smoking & $18(39.1)$ \\
\hline \multicolumn{2}{|l|}{ Pharmacological therapy } \\
\hline Aspirin & $28(22.8)$ \\
\hline Statins & $25(20.3)$ \\
\hline Beta blockers & $31(25.2)$ \\
\hline ACEI or ARB & $34(27.6)$ \\
\hline Insulin & $5(4.1)$ \\
\hline $\mathrm{EF}(\%)$ & $63(60-68)$ \\
\hline \multicolumn{2}{|l|}{ Culprit vessel } \\
\hline Left main & $4(3.3)$ \\
\hline Left anterior descending & $83(67.5)$ \\
\hline Left circumflex & $10(8.1)$ \\
\hline Right coronary artery & $26(21.1)$ \\
\hline CTNI (ng/ml) & $0.10(0.02-7.33)$ \\
\hline $\mathrm{BNP}(\mathrm{pg} / \mathrm{ml})$ & $57(35-340)$ \\
\hline
\end{tabular}

Values are expressed as the mean \pm standard deviation, median (interquartile range) or $\mathrm{n}(\%)$. ACS, acute coronary syndrome; ACEI, angiotensin-converting enzyme inhibitor; $\mathrm{ARB}$, angiotensin-receptor blocker; EF, ejection fraction; CHD, coronary heart disease; CTNI, cardiac troponin I; BNP, b-type natriuretic peptide. Normal ranges: CTNI (0-0.04 ng/ml); BNP (0-125 pg/ml).

and were compared using a non-parametric test. Univariate and multivariate logistic regression analyses were performed to assess independent predictors. All of the statistical calculations were performed using SPSS software version 22 (IBM Corp.). $\mathrm{P}<0.05$ was considered to indicate statistical significance.

\section{Results}

Patient information. In the present study, 123 patients (age, $36 \pm 7$ years; male $87.0 \%$, female $13 \%$ ) who underwent CAG and OCT were analyzed. Their baseline clinical characteristics and CAG data are presented in Table I. Cigarette smoking, hypertension, T2D, hypercholesterolemia and MetS were present in $54.5,51.2,17.9,12.2$ and $66.7 \%$ of the study population, respectively. The percentages of patients using aspirin, statins, beta-blockers, ACEIs or ARBs, and insulin were $22.8,20.3,25.2,27.6$ and $4.1 \%$, respectively. The percentages of smokers in the stable angina cohort vs. the ACS cohort 

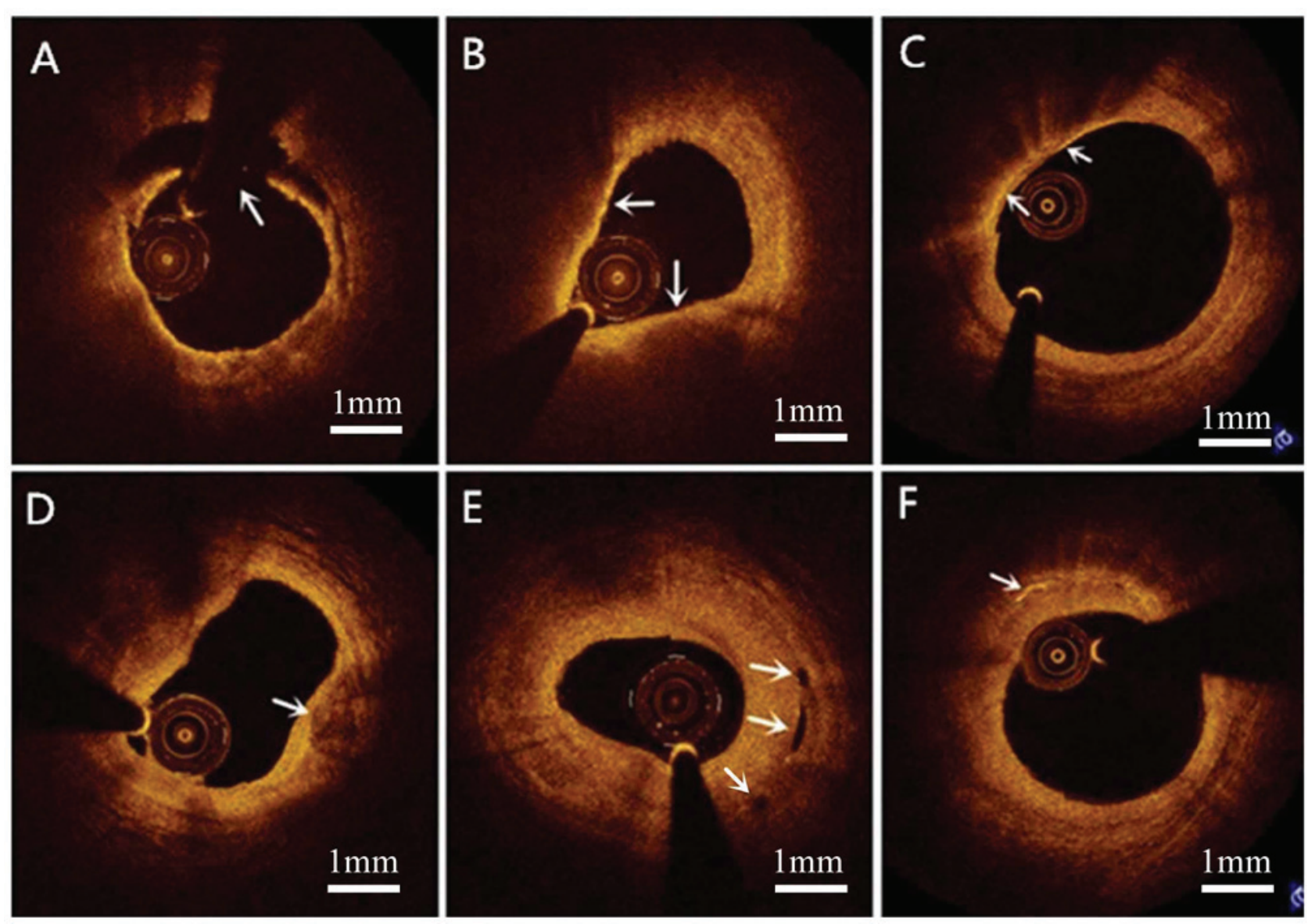

Figure 1. Representative optical coherence tomography images of different coronary plaque phenotypes (arrows). (A) Plaque rupture: The fibrous cap is broken and the plaque content is partially washed away, leaving a cavity (11 to 3 o'clock). (B) Thin-cap fibroatheroma: A large $\left(>90^{\circ}\right)$ lipidic core and a thin ( $\left.<65 \mu \mathrm{m}\right)$ fibrous cap (5 to 11 o'clock). (C) Macrophage accumulation: Signal-rich, distinct or confluent punctate regions that exceed the intensity of the background (9 to 12 o'clock). (D) Calcified nodule: A single region of calcium (signal-poor or heterogeneous, with a sharply delineated border) protruding into the lumen (3 to 4 o'clock). (E) Vasa vasorum: Small black holes within a plaque, 50-300 mm in diameter, present on at least 3 consecutive frames. (F) Cholesterol crystal: A thin linear structure with high backward scatter and without attenuation.

were $39.1 \%$ vs. $63.6 \%$, respectively $(\mathrm{P}=0.013)$. Left-anterior descending lesions accounted for $67.5 \%$ of all culprit lesions.

Characteristics of OCT-derived plaques and CVRFs. Distinct phenotypes of OCT-derived plaques and their associations with CVRFs are presented in Table II. TCFAs and macrophage accumulation were more prevalent in patients with than without MetS $(\mathrm{P}=0.020)$ and hypertension $(\mathrm{P}<0.001)$, respectively. Cholesterol crystals presented more frequently in patients with than without a family history of CHD $(\mathrm{P}=0.004)$ and hypercholesterolemia $(\mathrm{P}=0.031)$. The extent of plaque rupture was greater in smokers than in non-smokers $(\mathrm{P}=0.002)$. Vasa vasorum was more common in the culprit lesions of non-smokers than in those of smokers $(\mathrm{P}=0.003)$. By contrast, no significant association was observed between erosions and CVRFs or between calcified nodules and CVRFs in the present study. Representative OCT images are provided in Fig. 1.

Multivariate analysis. To assess the association between TFCAs and CVRFs or between plaque rupture and CVRFs, multivariate regression analyses were performed. Risk factors with $\mathrm{P}<0.100$ from the univariate analysis were included in the multivariate analyses. As presented in Table III, after adjusting for traditional confounding factors, MetS was independently associated with TCFAs [risk ratio (RR), 2.421; 95\% CI, 1.038-5.649; $\mathrm{P}=0.041]$. Of the CVRFs, smoking retained an independent association with plaque rupture (RR, 8.301; 95\% CI, 1.813-38.015; $\mathrm{P}=0.006$ ).

\section{Discussion}

The major results of the present study were as follows: i) MetS was independently associated with TCFAs; and ii) smoking was independently associated with plaque rupture. To the best of our knowledge, the present study was the first OCT study investigating the association between culprit plaque phenotype and CVRFs in young patients.

Young individuals with premature CHD may have fewer risk factors of CHD, but MetS is frequently present in this group of patients and puts them at a high risk of early-onset clinical CHD (16). In the present study, $66.7 \%$ of the patients had MetS. Kalantzi et al (7) reported that MetS is highly associated with ACS in patients $<45$ years of age and is more predictive than other cardiovascular risk factors. TCFAs are known as important predictors of cardiovascular events (CVEs) (17). Using virtual-histology intravascular ultrasound (VH-IVUS), Zheng et al (3) analyzed the volumetric plaque composition of the coronary arterial tree and its association 


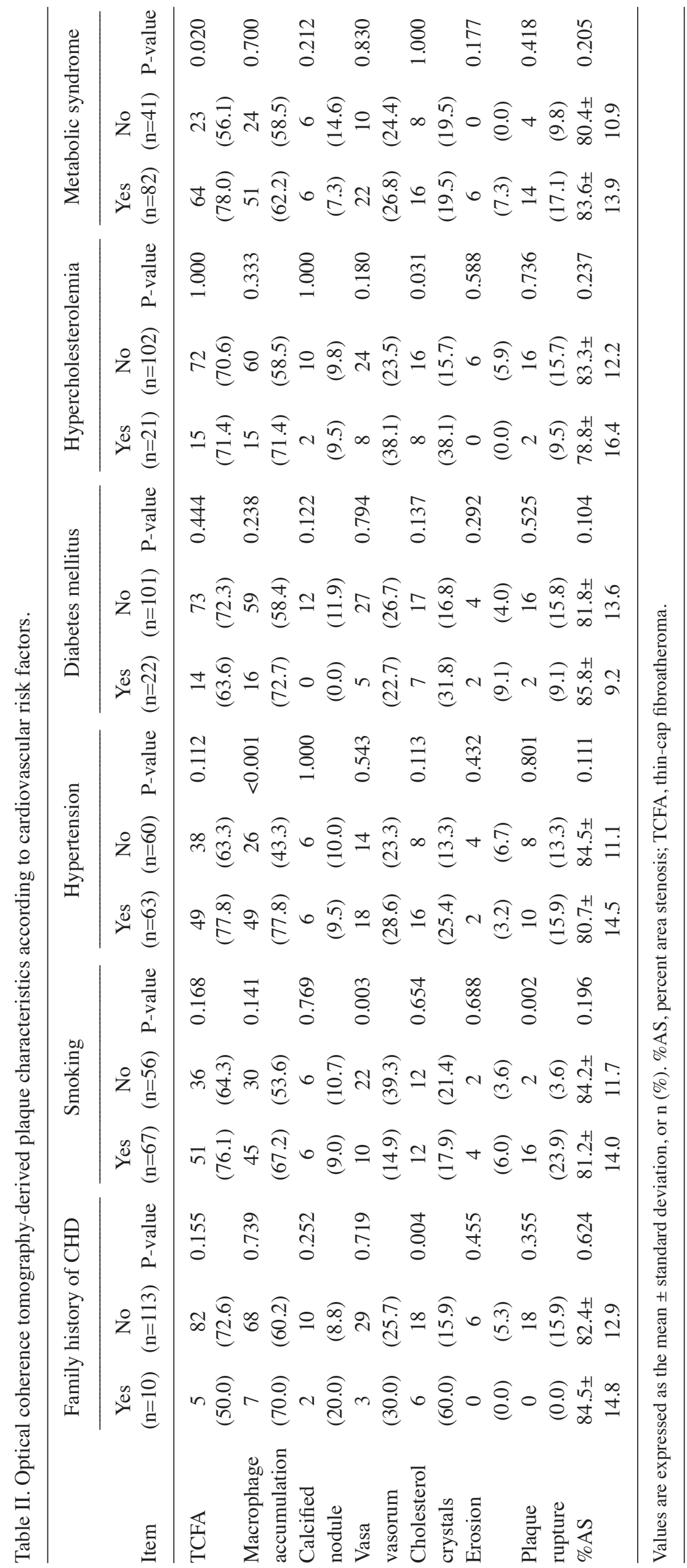


Table III. Univariate and multivariate analysis for TCFA and plaque rupture predictors.

A, Predictors of TCFA

\begin{tabular}{|c|c|c|c|c|c|c|}
\hline \multirow[b]{2}{*}{ Factor } & \multicolumn{3}{|c|}{ Univariate analysis } & \multicolumn{3}{|c|}{ Multivariate analysis } \\
\hline & RR & $95 \% \mathrm{CI}$ & P-value & RR & $95 \% \mathrm{CI}$ & P-value \\
\hline Gender & 1.54 & $0.514-4.610$ & 0.440 & & & \\
\hline Smoking & 1.771 & $0.809-3.877$ & 0.153 & & & \\
\hline Hypertension & 2.026 & $0.917-4.477$ & 0.081 & 1.574 & $0.679-3.650$ & 0.291 \\
\hline Diabetes mellitus & 0.671 & $0.254-1.774$ & 0.421 & & & \\
\hline Hypercholesterolemia & 1.042 & $0.369-2.942$ & 0.939 & & & \\
\hline Metabolic syndrome & 2.783 & $1.240-6.246$ & 0.013 & 2.421 & $1.038-5.649$ & 0.041 \\
\hline Aspirin & 0.678 & $0.277-1.660$ & 0.395 & & & \\
\hline Statins & 0.542 & $0.216-1.355$ & 0.190 & & & \\
\hline Beta blocker & 0.827 & $0.343-1.993$ & 0.673 & & & \\
\hline ACEI or ARB & 1.210 & $0.499-2.934$ & 0.674 & & & \\
\hline Insulin & 0.607 & $0.097-3.796$ & 0.594 & & & \\
\hline
\end{tabular}

$\mathrm{B}$, Predictors of plaque rupture

\begin{tabular}{|c|c|c|c|c|c|c|}
\hline \multirow[b]{2}{*}{ Factor } & \multicolumn{3}{|c|}{ Univariate analysis } & \multicolumn{3}{|c|}{ Multivariate analysis } \\
\hline & RR & $95 \% \mathrm{CI}$ & P-value & $\mathrm{RR}$ & $95 \% \mathrm{CI}$ & P-value \\
\hline Smoking & 8.471 & $1.855-38.690$ & 0.006 & 8.301 & $1.813-38.015$ & 0.006 \\
\hline Hypertension & 1.226 & $0.449-3.352$ & 0.691 & & & \\
\hline Diabetes mellitus & 0.531 & $0.113-2.499$ & 0.423 & & & \\
\hline Hypercholesterolemia & 0.566 & $0.120-2.670$ & 0.472 & & & \\
\hline Metabolic syndrome & 1.904 & $0.585-6.205$ & 0.285 & & & \\
\hline Aspirin & 0.380 & $0.082-1.763$ & 0.217 & & & \\
\hline Statins & 0.446 & $0.095-2.081$ & 0.304 & 2.064 & $0.422-10.093$ & 0.371 \\
\hline Beta blocker & 0.328 & $0.071-1.514$ & 0.153 & & & \\
\hline ACEI or ARB & 0.285 & $0.062-1.314$ & 0.107 & & & \\
\hline Insulin & 4.250 & $0.658-27.443$ & 0.128 & & & \\
\hline
\end{tabular}

TCFA, thin-cap fibroatheroma; ACEI, angiotensin-converting enzyme inhibitor; ARB, angiotensin-receptor blocker; RR, risk ratio.

with other CVRFs and MetS in patients diagnosed with isch-

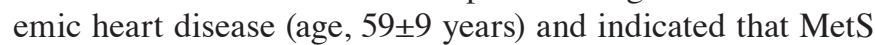
patients had more frequent VH-IVUS-derived TCFAs within the tree than non-MetS patients. Similarly, the present study suggested that patients with MetS had more frequent TCFAs than patients without MetS. Zheng et al (3) also demonstrated that T2D is independently associated with TCFAs.

Previous studies investigating features of coronary plaques in patients with MetS have provided conflicting results. Specifically, a previous IVUS study demonstrated no significant association between the presence of TCFAs and MetS in

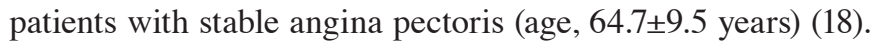
Another study using OCT indicated that coronary plaques

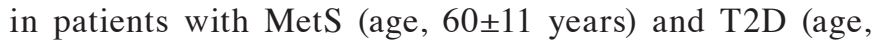
$59 \pm 11$ years) contain larger amounts of lipids, but neither MetS nor T2D was significantly associated with TCFAs (19). These conflicting results may have several reasons. First, the population of the present study was significantly younger than that in the aforementioned studies. The pathophysiology underlying atherosclerosis and plaque characteristics differ between young and old patients with CHD (20). Furthermore, in the study by Yonetsu et al (19), selected 198 patients who had nonculprit or nontarget coronary plaques with area stenosis $>50 \%$ as measured by OCT; however, whether they are culprit or non-culprit may affect the characteristics of plaques (21).

The present study indicated that cigarette smoking is independently associated with plaque rupture. Cigarette smoking is associated with a high incidence of CVEs, including ACS (22). It is also associated with a higher burden of necrotic cores in coronary atherosclerotic plaques, which may be one of the mechanisms underlying the increased risk it poses for plaque rupture and CVEs $(23,24)$. By far, the most common risk factor for early-onset CHD is cigarette smoking (6), which increases the risk of plaque rupture. Cigarette smokers accounted for $54.5 \%(n=67)$ of the patients of the present study. Cigarette smoking is thought to increase the burden of cardiovascular 
disease by inducing endothelial dysfunction, increasing the burden of coronary atherosclerosis and increasing the risk of plaque rupture and CVEs (25). T2D was not significantly associated with TCFAs or rupture in the present study. This may be due to the larger number of patients with T2D than without $(63.6 \%$ vs. $10.9 \%)$ using statins, which may reduce TCFAs and plaque rupture (26).

The universally acknowledged features of vulnerable plaques currently include TCFAs, macrophage accumulation, calcified nodules, vasa vasorum and cholesterol crystals (27-31). The association between TCFAs and CVRFs was described above. In the present study, macrophage accumulation was more common in patients with hypertension and cholesterol crystals were present more often in patients with a family history of CHD and hypercholesterolemia. By contrast, no significant correlation was observed between calcified nodules or vasa vasorum and CVRFs in the patients of the present study. The sample examined was not extracted from the general population but was rather composed of relatively young patients. Thus, the results may not be extrapolatable to the general population. In addition, the results may be affected by lifestyle factors, including diet and physical exercise.

In conclusion, by using OCT evaluation, the present study demonstrated that young patients with MetS had more extensive TCFAs and that young cigarette smokers were at increased risk for culprit plaque rupture. Young patients with CHD should therefore actively control their body weight, blood lipids, blood pressure and blood sugar levels, as well as quit smoking, so as to reduce the occurrence/risk of TCFAs and ruptures.

\section{Acknowledgements}

Not applicable.

\section{Funding}

No funding was received.

\section{Availability of data and materials}

The datasets used and/or analyzed during the present study are available from the corresponding author upon reasonable request.

\section{Authors' contributions}

YZ and JG conceived the study. FH, WL, YD and YL collected and analyzed the patients' general information. FH and WL wrote the manuscript. SY, XM and ZW analyzed the OCT images. All of the authors read and approved the final manuscript.

\section{Ethics approval and consent to participate}

The Ethics Committee of the Beijing Anzhen Hospital (Beijing, China) approved the study protocol and all of the participants provided written informed consent.

\section{Patient consent for publication}

All of the participants provided written informed consent for publication.

\section{Competing interests}

The authors declare that they have no competing interests.

\section{References}

1. Authors/Task Force Members: Piepoli MF, Hoes AW, Agewall S, Albus C, Brotons C, Catapano AL, Cooney M, Corrà U, Cosyns B, Deaton C, et al: 2016 European Guidelines on cardiovascular disease prevention in clinical practice: The Sixth Joint Task Force of the European Society of Cardiology and Other Societies on Cardiovascular Disease Prevention in Clinical Practice (constituted by representatives of 10 societies and by invited experts) Developed with the special contribution of the European Association for Cardiovascular Prevention \& Rehabilitation (EACPR). Atherosclerosis 252: 207-274, 2016.

2. Wilson PW, D'Agostino RB, Parise H, Sullivan L and Meigs JB: Metabolic syndrome as a precursor of cardiovascular disease and type 2 diabetes mellitus. Circulation 112: 3066-3072, 2005.

3. Zheng M, Choi SY, Tahk SJ, Lim HS, Yang HM, Choi BJ, Yoon MH, Park JS, Hwang GS and Shin JH: The relationship between volumetric plaque components and classical cardiovascular risk factors and the metabolic syndrome a 3-vessel coronary artery virtual histology-intravascular ultrasound analysis. JACC Cardiovasc Interv 4: 503-510, 2011.

4. Rivera JJ, Nasir K, Cox PR, Choi E, Yoon Y, Cho I, Chun EJ, Choi S, Blumenthal RS and Chang HJ: Association of traditional cardiovascular risk factors with coronary plaque sub-types assessed by 64-slice computed tomography angiography in a large cohort of asymptomatic subjects. Atherosclerosis 206: 451-457, 2009.

5. De Rosa R, Vasa-Nicotera M, Leistner DM, Reis SM, Thome CE, Boeckel J, Fichtlscherer S and Zeiher AM: Coronary atherosclerotic plaque characteristics and cardiovascular risk factors-insights from an optical coherence tomography study. Circ J 81: 1165-1173, 2017.

6. Aggarwal A, Srivastava S and Velmurugan M: Newer perspectives of coronary artery disease in young. World J Cardiol 8: 728-734, 2016

7. Kalantzi K, Korantzopoulos P, Tzimas P, Katsouras CS, Goudevenos JA and Milionis HJ: The relative value of metabolic syndrome and cardiovascular risk score estimates in premature acute coronary syndromes. Am Heart J 155: 534-540, 2008.

8. Rathore S, Terashima M, Matsuo H, Kinoshita Y, Kimura M, Tsuchikane E, Nasu K, Ehara M, Asakura Y, Katoh O and Suzuki T: Association of coronary plaque composition and arterial remodelling: A optical coherence tomography study. Atherosclerosis 221: 405-415, 2012.

9. Finn AV, Nakano M, Narula J, Kolodgie FD and Virmani R: Concept of vulnerable/unstable plaque. Arterioscler Thromb Vasc Biol 30: 1282-1292, 2010.

10. Jang IK, Bouma BE, Kang DH, Park SJ, Park SW, Seung KB, Choi KB, Shishkov M, Schlendorf K, Pomerantsev E, et al: Visualization of coronary atherosclerotic plaques in patients using optical coherence tomography: Comparison with intravascular ultrasound. J Am Coll Cardiol 39: 604-609, 2002.

11. Alberti KG, Eckel RH, Grundy SM, Zimmet PZ, Cleeman JI, Donato KA, Fruchart JC, James WP, Loria CM, Smith SC Jr, et al: Harmonizing the Metabolic Syndrome: A Joint Interim Statement of the International Diabetes Federation Task Force on Epidemiology and Prevention; National Heart, Lung, and Blood Institute; American Heart Association; World Heart Federation; International Atherosclerosis Society; and International Association for the Study of Obesity. Circulation 120: 1640-1645, 2009.

12. Jia H, Dai J, Hou J, Xing L, Ma L, Liu H, Xu M, Yao Y, Hu S, Yamamoto E, et al: Effective anti-thrombotic therapy without stenting: Intravascular optical coherence tomography-based management in plaque erosion (the EROSION study). Eur Heart J 38: 792-800, 2017.

13. Tian J, Hou J, Xing L, Kim SJ, Yonetsu T, Kato K, Lee H, Zhang S, Yu B and Jang IK: Significance of intraplaque neovascularisation for vulnerability: Optical coherence tomography study. Heart 98 : 1504-1509, 2012.

14. Nishimura S, Ehara S, Hasegawa T, Matsumoto K, Yoshikawa J and Shimada K: Cholesterol crystal as a new feature of coronary vulnerable plaques: An optical coherence tomography study. J Cardiol 69: 253-259, 2017 
15. Tearney GJ, Regar E, Akasaka T, Adriaenssens T, Barlis P, Bezerra HG, Bouma B, Bruining N, Cho JM, Chowdhary S, et al: Consensus standards for acquisition, measurement, and reporting of intravascular optical coherence tomography studies: A report from the International Working Group for Intravascular Optical Coherence Tomography Standardization and Validation. J Am Coll Cardiol 59: 1058-1072, 2012.

16. Iribarren C, Go AS, Husson G, Sidney S, Fair JM, Quertermous T, Hlatky MA and Fortmann SP: Metabolic syndrome and early-onset coronary artery disease: Is the whole greater than its parts? J Am Coll Cardiol 48: 1800-1807, 2006.

17. Iannaccone M, Quadri G, Taha S, D'Ascenzo F, Montefusco A, Omede P, Jang IK, Niccoli G, Souteyrand G, Yundai C, et al: Prevalence and predictors of culprit plaque rupture at OCT in patients with coronary artery disease: A meta-analysis. Eur Heart J Cardiovasc Imaging 17: 1128-1137, 2016.

18. Lee MG, Jeong MH, Kim DH, Lee KH, Park KH, Sim DS, Yoon NS, Yoon HJ, Kim KH, Park HW, et al: Can metabolic syndrome predict the vulnerable plaque in patients with stable angina pectoris? Virtual histology-intravascular ultrasound analysis. J Cardiol 59: 266-274, 2012.

19. Yonetsu T, Kato K, Uemura S, Kim BK, Jang Y, Kang SJ, Park SJ, Lee S, Kim SJ, Jia H, et al: Features of coronary plaque in patients with metabolic syndrome and diabetes mellitus assessed by 3 -vessel optical coherence tomography. Circ Cardiovase Imaging 6: 665-673, 2013.

20. Barbero U, Scacciatella P, Iannaccone M, D'Ascenzo F, Niccoli G, Colombo F, Ugo F, Colangelo S, Mancone M, Calcagno S, et al: Culprit plaque characteristics in younger versus older patients with acute coronary syndromes: An optical coherence tomography study from the FORMIDABLE registry. Catheter Cardiovasc Interv 92: E1-E8, 2018.

21. Trusinskis K, Juhnevica D, Strenge K and Erglis A: iMap intravascular ultrasound evaluation of culprit and non-culprit lesions in patients with ST-elevation myocardial infarction. Cardiovasc Revasc Med 14: 71-75, 2013.

22. Erhardt L: Cigarette smoking: An undertreated risk factor for cardiovascular disease. Atherosclerosis 205: 23-32, 2009.
23. Abtahian F, Yonetsu T, Kato K, Jia H, Vergallo R, Tian J, Hu S, McNulty I, Lee H, Yu B and Jang IK: Comparison by optical coherence tomography of the frequency of lipid coronary plaques in current smokers, former smokers, and nonsmokers. Am J Cardiol 114: 674-680, 2014.

24. Bolorunduro O,Cushman C, Kapoor D, Alexander K, Cuellar-Silva J, Giri S, Robinson V and Ibebuogu UN: Comparison of coronary atherosclerotic plaque burden and composition of culprit lesions between cigarette smokers and non-smokers by in vivo virtual histology intravascular ultrasound. J Invasive Cardiol 27: 354-358, 2015.

25. Csordas A and Bernhard D: The biology behind the atherothrombotic effects of cigarette smoke. Nat Rev Cardiol 10: 219-230, 2013.

26. Gili S, Iannaccone M, Colombo F, Montefusco A, Amabile N, Calcagno S, Capodanno D, Scalone G, Rognoni A, Omedè $\mathrm{P}$, et al: Effects of statins on plaque rupture assessed by optical coherence tomography in patients presenting with acute coronary syndromes: Insights from the optical coherence tomography (OCT)-FORMIDABLE registry. Eur Heart J Cardiovase Imaging 19: 524-531, 2018.

27. Kume T and Uemura S: Current clinical applications of coronary optical coherence tomography. Cardiovasc Interv Ther 33: 1-10, 2018.

28. Lee T, Mintz GS, Matsumura M, Zhang W, Cao Y, Usui E, Kanaji Y, Murai T, Yonetsu T, Kakuta T and Maehara A: Prevalence, predictors, and clinical presentation of a calcified nodule as assessed by optical coherence tomography. JACC Cardiovasc Imaging 10: 883-891, 2017.

29. Gonzalez L and Trigatti BL: Macrophage apoptosis and necrotic core development in atherosclerosis: A rapidly advancing field with clinical relevance to imaging and therapy. Can J Cardiol 33: 303-312, 2017.

30. Janoudi A, ShamounFE,Kalavakunta JK and Abela GS: Cholesterol crystal induced arterial inflammation and destabilization of atherosclerotic plaque. Eur Heart J 37: 1959-1967, 2016.

31. Taruya A, Tanaka A, Nishiguchi T, Matsuo Y, Ozaki Y, Kashiwagi M, Shiono Y, Orii M, Yamano T, Ino Y, et al: Vasa vasorum restructuring in human atherosclerotic plaque vulnerability: A clinical optical coherence tomography study. J Am Coll Cardiol 65: 2469-2477, 2015. 